\title{
Global Behavior of a Rational Difference Equation with Quadratic Term
}

\author{
R. ABo-ZEID
}

Abstract. In this paper, we determine the forbidden set, introduce an explicit formula for the solutions and discuss the global behavior of all solutions of the difference equation

$$
x_{n+1}=\frac{a x_{n} x_{n-1}}{b x_{n}-c x_{n-2}}, \quad n=0,1, \ldots
$$

where $a, b, c$ are positive real numbers and the initial conditions $x_{-2}, x_{-1}, x_{0}$ are real numbers.

\section{INTRODUCTION}

Li and Zhu [10] discussed the global asymptotic stability of the difference equation

$$
x_{n+1}=\frac{x_{n} x_{n-1}+a}{x_{n}+x_{n-1}}, \quad n=0,1, \ldots
$$

where $a \in[0, \infty)$ and $x_{-1}, x_{0}$ are positive real numbers.

In [6] H. Sedaghat determined the global behavior of all solutions of the rational difference equations

$$
x_{n+1}=\frac{a x_{n-1}}{x_{n} x_{n-1}+b}, \quad x_{n+1}=\frac{a x_{n} x_{n-1}}{x_{n}+b x_{n-2}}, \quad n=0,1, \ldots
$$

where $a, b>0$.

In this paper, we derive the forbidden set, introduce an explicit formula for the solutions and discuss the global behavior of all solutions of the difference equation

$$
x_{n+1}=\frac{a x_{n} x_{n-1}}{b x_{n}-c x_{n-2}}, \quad n=0,1, \ldots
$$

where $a, b, c$ are positive real numbers and the initial conditions $x_{-2}, x_{-1}, x_{0}$ are real numbers.

2010 Mathematics Subject Classification. Primary: 39A20, 39A21, 39A23, 39A30.

Key words and phrases. difference equation, periodic solution, unbounded solution. 


\section{Forbidden Set and Solutions of Qquation (1.1)}

In this section we derive the forbidden set and introduce an explicit formula for the solutions of the difference equation (1.1).

Suppose that $x_{0} x_{-1}=0$. Then we have the following:

If $x_{0}=0$ and $x_{-1} \neq 0$, then $x_{3}$ is undefined.

If $x_{-1}=0$ and $x_{0} \neq 0$, then $x_{2}$ is undefined.

If $x_{-2}=0$ and $x_{0} x_{-1} \neq 0$, then $x_{1}=\frac{a}{b} x_{-1} \neq 0$.

Therefore, we can start with the nonzero initial conditions $x_{-1}, x_{0}, x_{1}$, which we shall discuss.

Now suppose that $x_{-i} \neq 0$ for all $i=0,1,2$. Using the substitution $r_{n}=\frac{x_{n}}{x_{n-1}}$, equation (1.1) becomes

$$
r_{n+1}=\frac{a r_{n-1}}{b r_{n} r_{n-1}-c}, \quad n=0,1, \ldots
$$

Now using the substitution $l_{n}=\frac{1}{r_{n} r_{n-1}}$, we can obtain the linear nonhomogeneous difference equation

$$
l_{n+1}=-\frac{c}{a} l_{n}+\frac{b}{a}, \quad l_{0}=\frac{1}{r_{0} r_{-1}}=\frac{1}{\alpha}, \quad n=0,1, \ldots
$$

The solution of equation (2.2) is

$$
\begin{aligned}
l_{n} & =\left(-\frac{c}{a}\right)^{n} l_{0}+\frac{b}{a} \sum_{i=0}^{n-1}\left(-\frac{c}{a}\right)^{i} \\
& =\frac{\left(-\frac{c}{a}\right)^{n} a+b \alpha \sum_{i=0}^{n-1}\left(-\frac{c}{a}\right)^{i}}{a \alpha}, \quad n=0,1, \ldots
\end{aligned}
$$

But $l_{n}=\frac{1}{r_{n} r_{n-1}}=\frac{x_{n-2}}{x_{n}}$. Therefore,

$$
\frac{x_{n}}{x_{n-2}}=\frac{a \alpha}{\left(-\frac{c}{a}\right)^{n} a+b \alpha \sum_{i=0}^{n-1}\left(-\frac{c}{a}\right)^{i}}, \quad n=0,1, \ldots
$$

When $n=n_{0}$ for some $n_{0} \in \mathbb{N}$, if we set $\alpha=\frac{c}{b \sum_{l=0}^{n_{0}}\left(-\frac{a}{c}\right)^{l}}$ in equation (2.4), we obtain

$$
\frac{x_{n}}{x_{n-2}}=\frac{c}{b}
$$

Therefore, $y_{n_{0}+1}$ is undefined.

On the other hand, from equation (1.1) we have that

$$
\frac{x_{n-1}}{x_{n-3}}=\frac{c x_{n} / x_{n-2}}{b x_{n} / x_{n-2}-a}
$$

For a fixed $n_{0} \in \mathbb{N}$, suppose that we have $y_{n_{0}+1}$ is undefined. This implies that

$$
b x_{n_{0}}-c x_{n_{0}-2}=0
$$


That is

$$
\frac{x_{n_{0}}}{x_{n_{0}-2}}=\frac{c}{b} .
$$

Hence using equation (2.4), we have the following:

$$
\begin{gathered}
\frac{x_{n_{0}-1}}{x_{n_{0}-3}}=\frac{c^{2}}{b(c-a)}=\frac{c}{b \sum_{i=0}^{1}\left(-\frac{a}{c}\right)^{i}}, \\
\frac{x_{n_{0}-2}}{x_{n_{0}-4}}=\frac{c \frac{c^{2}}{b(c-a)}}{b \frac{c^{2}}{b(c-a)}-a}=\frac{c}{b \sum_{i=0}^{2}\left(-\frac{a}{c}\right)^{i}} .
\end{gathered}
$$

Now suppose that

$$
\frac{x_{n_{0}-(k-1)}}{x_{n_{0}-(k+1)}}=\frac{c}{b \sum_{i=0}^{k-1}\left(-\frac{a}{c}\right)^{i}}, \quad 0 \leq k \leq n_{0} .
$$

Then

$$
\begin{aligned}
\frac{x_{n_{0}-(k)}}{x_{n_{0}-(k+2)}} & =\frac{c \frac{c}{b \sum_{i=0}^{k-1}\left(-\frac{a}{c}\right)^{i}}}{b \frac{c}{b \sum_{i=0}^{k-1}\left(-\frac{a}{c}\right)^{i}}-a}=\frac{c}{b} \frac{c}{c-a \sum_{i=0}^{k-1}\left(-\frac{a}{c}\right)^{i}} \\
& =\frac{c}{b} \frac{1}{1+\sum_{i=0}^{k-1}\left(-\frac{a}{c}\right)^{i+1}}=\frac{c}{b \sum_{i=0}^{k}\left(-\frac{a}{c}\right)^{i}} .
\end{aligned}
$$

Therefore, for $k=n_{0}$ we have

$$
\frac{x_{0}}{x_{-2}}=\alpha=\frac{c}{b \sum_{i=0}^{n_{0}}\left(-\frac{a}{c}\right)^{i}} .
$$

These observations lead us to conclude the following result.

Proposition 2.1. The forbidden set $F$ of equation (1.1) is

$$
\begin{aligned}
F=\bigcup_{n=0}^{\infty}\left\{\left(u_{0}, u_{-1}, u_{-2}\right): u_{0}\right. & \left.=u_{-2}\left(\frac{c}{b \sum_{i=0}^{n}\left(\frac{-a}{c}\right)^{i}}\right)\right\} \cup \\
& \left\{\left(u_{0}, u_{-1}, u_{-2}\right): u_{0}=0\right\} \cup\left\{\left(u_{0}, u_{-1}, u_{-2}\right): u_{-1}=0\right\} .
\end{aligned}
$$

Using equation (2.4), we obtain the following result.

Theorem 2.2. Let $x_{-2}, x_{-1}$ and $x_{0}$ be real numbers such that $\left(x_{0}, x_{-1}, x_{-2}\right) \notin$ $F$. If $a \neq c$, then the solution $\left\{x_{n}\right\}_{n=-2}^{\infty}$ of equation (1.1) is

$$
x_{n}= \begin{cases}x_{-1} \prod_{j=0}^{\frac{n-1}{2}} \frac{a+c}{\theta(a+c)\left(-\frac{c}{a}\right)^{2 j+1}+b}, & n=1,3,5, \ldots \\ x_{0} \prod_{j=0}^{\frac{n-2}{2}} \frac{a+c}{\theta(a+c)\left(-\frac{c}{a}\right)^{2 j+2}+b}, & n=2,4,6, \ldots\end{cases}
$$

where $\theta=\frac{a+c-b \alpha}{\alpha(a+c)}$. 


\section{Global Behavior of Equation (1.1)}

In this section, we investigate the global behavior of equation (1.1) with $a \neq c$, using the explicit formula of its solution.

We can write the solution of equation (1.1) in the form:

$$
x_{2 m+i}=x_{-2+i} \prod_{j=0}^{m} \beta_{i}(j), \quad i=1,2 \text { and } m=0,1, \ldots
$$

where

$$
\beta_{i}(j)=\frac{a+c}{\theta(a+c)\left(-\frac{c}{a}\right)^{2 j+i}+b}, \quad i=1,2 .
$$

Theorem 3.1. Let $\left\{x_{n}\right\}_{n=-2}^{\infty}$ be a solution of equation (1.1) such that $\left(x_{0}, x_{-1}, x_{-2}\right) \notin F$. Then the following statements are true.

(1) If $a<c$, then $\left\{x_{n}\right\}_{n=-2}^{\infty}$ converges to zero.

(2) If $a>c$, then we have the following:

(a) If $\frac{a+c}{b}<1$, then $\left\{x_{n}\right\}_{n=-2}^{\infty}$ converges to zero.

(b) If $\frac{a+c}{b}>1$, then both $\left\{x_{2 n}\right\}_{n=-1}^{\infty}$ and $\left\{x_{2 n+1}\right\}_{n=-1}^{\infty}$ are unbounded.

Proof.

(1) If $a<c$, then $\beta_{i}(j)$ converges to 0 as $j \rightarrow \infty, i=1,2$. It follows that, there exists $j_{0} \in \mathbb{N}$ such that, $\left|\beta_{i}(j)\right|<\mu$, with some $0<\mu<1$ for all $j \geq j_{0}$. Therefore,

$$
\begin{aligned}
\left|x_{2 m+i}\right| & =\left|x_{-2+i}\right|\left|\prod_{j=0}^{m} \beta_{i}(j)\right| \\
& =\left|x_{-2+i}\right|\left|\prod_{j=0}^{j_{0}-1} \beta_{i}(j)\right|\left|\prod_{j=j_{0}}^{m} \beta_{i}(j)\right| \\
& <\left|x_{-2+i}\right|\left|\prod_{j=0}^{j_{0}-1} \beta_{i}(j)\right| \mu^{m-j_{0}+1}
\end{aligned}
$$

As $m$ tends to infinity, the solution $\left\{x_{n}\right\}_{n=-2}^{\infty}$ converges to zero.

(2) Suppose that $a>c$. Then we have the following:

(a) If $\frac{a+c}{b}<1$, then $\beta_{i}(j)$ converges to $\frac{a+c}{b}<1$ as $j \rightarrow \infty, i=1,2$. Therefore, there exists $j_{1} \in \mathbb{N}$ such that, $0<\beta_{i}(j)<\mu_{1}$, with some $0<\mu_{1}<1$ for all $j \geq j_{1}$ and the solution $\left\{x_{n}\right\}_{n=-2}^{\infty}$ converges to zero as in (1).

(b) If $\frac{a+c}{b}>1$, then $\beta_{i}(j)$ converges to $\frac{a+c}{b}>1$ as $j \rightarrow \infty, i=1,2$. Then there exists $j_{0} \in \mathbb{N}$ such that, $\beta_{i}(j)>\nu$, for some $\nu>1$ for all $j \geq j_{0}$. 
Hence

$$
\begin{aligned}
\left|x_{2 m+i}\right| & =\left|x_{-2+i}\right|\left|\prod_{j=0}^{m} \beta_{i}(j)\right| \\
& =\left|x_{-2+i}\right|\left|\prod_{j=0}^{j_{0}-1} \beta_{i}(j)\right| \prod_{j=j_{0}}^{m} \beta_{i}(j) \\
& >\left|x_{-2+i}\right|\left|\prod_{j=0}^{j_{0}-1} \beta_{i}(j)\right| \nu^{m-j_{0}+1} .
\end{aligned}
$$

Therefore, both of the subsequences $\left\{x_{2 n}\right\}_{n=-1}^{\infty}$ and $\left\{x_{2 n+1}\right\}_{n=-1}^{\infty}$ are unbounded.

\section{CASE $a+c=b$}

In order to discuss the case when $a+c=b$ with $a>c$, we need to remember the behavior of equation (2.1) with $a+c=b$ with $a>c$.

Many authors [1-9] discussed the behavior of the solutions of some special cases of the equation

$$
x_{n+1}=\frac{A x_{n-1}}{B+C x_{n} x_{n-1}}, \quad n=0,1, \ldots
$$

where $A, B, C$ are real numbers, but with reducing the numbers of parameters to one or two.

We shall derive only some results concern the behavior of the solutions of equation (2.1), that we shall use.

The following theorem gives the solution of equation (2.1).

Theorem 4.1. Let $r_{-1}, r_{0}$ be real numbers such that $r_{-1} r_{0}=\alpha \neq \frac{c}{b \sum_{i=0}^{n}\left(-\frac{a}{c}\right)^{i}}$ for any $n \in \mathbb{N}$. Then the solution of equation (2.1) is

$$
r_{n}= \begin{cases}r_{-1} \prod_{j=0}^{\frac{n-1}{2}} \frac{\theta(a+c)\left(-\frac{c}{a}\right)^{2 j}+b}{\theta(a+c)\left(-\frac{c}{a}\right)^{2 j+1}+b}, & n=1,3,5, \ldots \\ r_{0} \prod_{j=0}^{\frac{n-2}{2}} \frac{\theta(a+c)\left(-\frac{c}{a}\right)^{2 j+1}+b}{\theta(a+c)\left(-\frac{c}{a}\right)^{2 j+2}+b}, & n=2,4,6, \ldots\end{cases}
$$

where $\theta=\frac{a+c-b \alpha}{\alpha(a+c)}$.

The solution of equation (2.1) can be written as:

$$
r_{2 m+i}=r_{-2+i} \prod_{j=0}^{m} \gamma_{i}(j), \quad i=1,2 \text { and } m=0,1, \ldots
$$

where

$$
\gamma_{i}(j)=\frac{\theta(a+c)\left(-\frac{c}{a}\right)^{2 j+i-1}+b}{\theta(a+c)\left(-\frac{c}{a}\right)^{2 j+i}+b}, \quad i=1,2 .
$$


Now assume that $a+c=b$ and $\alpha \neq 0$. Then we have

$$
\gamma_{i}(j)=\frac{\theta\left(-\frac{c}{a}\right)^{2 j+i-1}+1}{\theta\left(-\frac{c}{a}\right)^{2 j+i}+1}, \quad i=1,2 .
$$

Theorem 4.2. Assume that $a>c$ and let $\left\{r_{n}\right\}_{n=-1}^{\infty}$ be a solution of equation (2.1) such that $\alpha \neq 1$ and $r_{-1} r_{0}=\alpha \neq \frac{c}{b \sum_{i=0}^{n}\left(-\frac{a}{c}\right)^{2}}$ for any $n \in N$. Then $\left\{r_{2 m+1}\right\}_{m=-1}^{\infty}$ and $\left\{r_{2 m}\right\}_{m=-1}^{\infty}$ converge to finite limits.

Proof. Let $\left\{r_{n}\right\}_{n=-1}^{\infty}$ is a solution of equation (2.1) such that $r_{-1} r_{0}=\alpha \neq$ $\frac{c}{b \sum_{i=0}^{n}\left(-\frac{a}{c}\right)^{i}}$ for any $n \in N$.

The condition $\alpha \neq 1$ (where $\frac{a+c}{b}=1$ ) ensures that the solution $\left\{r_{n}\right\}_{n=-1}^{\infty}$ is not a period-2 solution.

We claim that, there exists $j_{0} \in \mathbb{N}$ such that $\gamma_{i}(j)>0$ for all $j \geq j_{0}$.

For, let $a_{i}(j)=\theta\left(-\frac{c}{a}\right)^{2 j+i-1}+1$ and $b_{i}(j)=\theta\left(-\frac{c}{a}\right)^{2 j+i}+1$. Then we can write

$$
\gamma_{i}(j)=\frac{\theta\left(-\frac{c}{a}\right)^{2 j+i-1}+1}{\theta\left(-\frac{c}{a}\right)^{2 j+i}+1}=\frac{a_{i}(j)}{b_{i}(j)}, \quad i=1,2 .
$$

We have the following situations:

- If $\theta<0$, then, we have the following:

- If $i=1$, then $b_{1}(j)>0$ for all $j \in \mathbb{N}$. But as $a_{1}(j)$ converges to 1 , there exists $j_{1} \in \mathbb{N}$ such that $a_{1}(j)>0$ for all $j \geq j_{1}$.

Therefore, $\gamma_{1}(j)=\frac{a_{1}(j)}{b_{1}(j)}>0$ for all $j \geq j_{1}$.

- If $i=2$, then $a_{2}(j)>0$ for all $j \in \mathbb{N}$. But as $b_{2}(j)$ converges to 1 , there exists $j_{2} \in \mathbb{N}$ such that $b_{2}(j)>0$ for all $j \geq j_{2}$.

Therefore, $\gamma_{2}(j)=\frac{a_{2}(j)}{b_{2}(j)}>0$ for all $j \geq j_{2}$.

In all cases, there exists a natural number $j_{0}=\max \left\{j_{1}, j_{2}\right\}$ such that $\gamma_{i}(j)=\frac{a_{i}(j)}{b_{i}(j)}>0, i=1,2$ for all $j \geq j_{0}$.

- If $\theta>0$, the situation is similar and will be omitted.

Claim is complete.

Now for each $i \in\{1,2\}$, we have for large $m$

$$
\begin{aligned}
r_{2 m+i} & =r_{-2+i} \prod_{j=0}^{m} \frac{\theta\left(-\frac{c}{a}\right)^{2 j+i-1}+1}{\theta\left(-\frac{c}{a}\right)^{2 j+i}+1} \\
& =r_{-2+i} \prod_{j=0}^{m} \gamma_{i}(j) \\
& =r_{-2+i} \prod_{j=0}^{j_{0}-1} \gamma_{i}(j) \prod_{j=j_{0}}^{m} \gamma_{i}(j) \\
& =r_{-2+i} \prod_{j=0}^{j_{0}-1} \gamma_{i}(j) \exp \left(\sum_{j=j_{0}}^{m} \ln \gamma_{i}(j)\right)
\end{aligned}
$$


We shall test the convergence of the series $\sum_{j=j_{0}}^{\infty}\left|\ln \gamma_{i}(j)\right|$.

Since $\lim _{j \rightarrow \infty}\left|\frac{\ln \gamma_{i}(j+1)}{\ln \gamma_{i}(j)}\right|=\frac{0}{0}$, using L'Hospital's rule we obtain

$$
\lim _{j \rightarrow \infty}\left|\frac{\ln \gamma_{i}(j+1)}{\ln \gamma_{i}(j)}\right|=\left(\frac{c}{a}\right)^{2}<1 .
$$

Then from d'Alembert's test that the series $\sum_{j=j_{0}}^{\infty}\left|\ln \gamma_{i}(j)\right|$ is convergent, it follows that there exist 2 real numbers $\rho_{i} \in \mathbb{R}$ such that

$$
\lim _{m \rightarrow \infty} r_{2 m+i}=\rho_{i}, \quad i \in\{0,1\} .
$$

Now we are ready to introduce the main results in this section.

Theorem 4.3. Assume that $\left\{x_{n}\right\}_{n=-2}^{\infty}$ is a solution of equation (1.1) such that $\left(x_{0}, x_{-1}, x_{-2}\right) \notin F$ and let $a+c=b$. If $\alpha=1$, then $\left\{x_{n}\right\}_{n=-2}^{\infty}$ is eventually periodic solution with period 2 .

Proof. Assume that $a+c=b$. If $\alpha=1$, then $\theta=0$. Therefore,

$$
\begin{aligned}
x_{2 m+i} & =x_{-2+i} \prod_{j=0}^{m} \frac{a+c}{\theta(a+c)\left(-\frac{c}{a}\right)^{2 j+i}+b} \\
& =x_{-2+i}, \quad i=1,2 \text { and } m=0,1, \ldots
\end{aligned}
$$

Theorem 4.4. Assume that $\left\{x_{n}\right\}_{n=-2}^{\infty}$ is a solution of equation (1.1) such that $\left(x_{0}, x_{-1}, x_{-2}\right) \notin F$ and let $a+c=b$. If $\alpha \neq 1$, then we have the following:

(1) If $a<c$, then $\left\{x_{n}\right\}_{n=-2}^{\infty}$ converges to zero.

(2) If $a>c$, then $\left\{x_{n}\right\}_{n=-2}^{\infty}$ converges to a period-2 solution $\left\{\mu_{0}, \mu_{1}\right\}$ such that $\mu_{1}=\mu_{0} \rho_{1}$, where $\rho_{1}$ is as in Theorem (4.2).

Proof.

(1) The proof is similar to that in theorem (3.1).

(2) Suppose that $a+c=b$ and $a>c$, then $\beta_{i}(j)=\frac{1}{\theta\left(-\frac{c}{a}\right)^{2 j+i}+1}$ converges to $1, i=1,2$.

By an argument similar to that in theorem (4.2), there exists $j_{0} \in \mathbb{N}$ such that, $\beta_{i}(j)>0$, for all $j \geq j_{0}$.

Hence

$$
\begin{aligned}
x_{2 m+i} & =x_{-2+i} \prod_{j=0}^{m} \beta_{i}(j)=x_{-2+i} \prod_{j=0}^{j_{0}-1} \beta_{i}(j) \prod_{j=j_{0}}^{m} \beta_{i}(j) \\
& =x_{-2+i} \prod_{j=0}^{j_{0}-1} \beta_{i}(j) \exp \left(\sum_{j=j_{0}}^{m} \ln \beta_{i}(j)\right) .
\end{aligned}
$$

We shall test the convergence of the series $\sum_{j=j_{0}}^{\infty}\left|\ln \beta_{i}(j)\right|$. 
Since $\lim _{j \rightarrow \infty}\left|\frac{\ln \beta_{i}(j+1)}{\ln \beta_{i}(j)}\right|=\frac{0}{0}$, using L'Hospital's rule we obtain

$$
\lim _{j \rightarrow \infty}\left|\frac{\ln \beta_{i}(j+1)}{\ln \beta_{i}(j)}\right|=\left(\frac{c}{a}\right)^{2}<1 .
$$

It follows from D'Alemberts' test that the series $\sum_{j=j_{0}}^{\infty}\left|\ln \beta_{i}(j)\right|$ is convergent.

This ensures that there are two real numbers $\mu_{0}, \mu_{1}$ such that

$$
\lim _{m \rightarrow \infty} x_{2 m+i}=\mu_{i}, \quad i \in\{0,1\} .
$$

Moreover, as $x_{2 m+1}=x_{2 m} r_{2 m+1}$, then $\mu_{1}=\mu_{0} \rho_{1}$ where

$$
\rho_{1}=r_{-1} \prod_{j=0}^{\infty} \frac{\theta\left(-\frac{c}{a}\right)^{2 j}+1}{\theta\left(-\frac{c}{a}\right)^{2 j+1}+1} \quad \text { and } \quad \mu_{0}=x_{0} \prod_{j=1}^{\infty} \frac{1}{\theta\left(-\frac{c}{a}\right)^{2 j}+1} \text {. }
$$

\section{REFERENCES}

[1] M. Aloqeili, Dynamics of a rational difference equation, Appl. Math. Comput., 176 (2006), 768-774.

[2] C. Cinar, On the positive solutions of the difference equation $x_{n+1}=\frac{x_{n-1}}{1+x_{n} x_{n-1}}$, Appl. Math. Comput., 150 (2004), 21-24.

[3] C. Cinar, on the positive solutions of the difference equation $x_{n+1}=\frac{a x_{n-1}}{1+b x_{n} x_{n-1}}$, Appl. Math. Comput., 156 (2004), 587-590.

[4] C. Cinar, On the difference equation $x_{n+1}=\frac{x_{n-1}}{-1+x_{n} x_{n-1}}$, Appl. Math. Comput., 158 (2004), 813-816.

[5] S. Stevic, More on a rational recurrence relation, Appl. Math. E-Notes, 4 (2004), $80-84$.

[6] H. Sedaghat, Global behaviours of rational difference equations of orders two and three with quadratic terms, J. Diff. Eq. Appl., 15(3) (2009), 215-224.

[7] A. Andruch-Sobi lo, M. Migda, Further properties of the rational rercursive sequence $x_{n+1}=\frac{a x_{n-1}}{b+c x_{n} x_{n-1}}$, Opuscula Math., 26(3) (2006), 387-394.

[8] A. Andruch-Sobi lo, M. Migda, On the rational recursive sequence $x_{n+1}=\frac{a x_{n-1}}{b+c x_{n} x_{n-1}}$, Tatra Mt. Math. Publ., 43 (2009), 1-9.

[9] I. Bajo and E. Liz, Global behaviour of a second-order nonlinear difference equation, J. Diff. Eq. Appl., First published on 01 February 2011, DOI: $10.1080 / 10236191003639475$.

[10] X. Li, D. Zhu , Global asymptotic stability in a rational equation, J. Diff. Equations Appl., 9 (2003), 833-839.

\section{R. Abo-Zeid}

Department of Basic Science

High Institute for Engineering \& Modern Technology

CAIRO

EGYPT

E-mail address: abuzead73@yahoo.com 